\title{
Structural Proteomics: Methods in Deriving Protein Structural Information and Issues in Data Management
}

\author{
Shankari E. Mylvaganam, Muthuchidambram Prabhakaran, Suresh S. Tudor, Saied Moezzi, \\ and Kalyanaraman Ramnarayan \\ Structural Bioinformatics, San Diego, CA, USA
}

Computational Proteomics Supplement 32:S42-S46 (March 2002)

\section{ABSTRACT}

Structural proteomics is an emerging paradigm that is gaining importance in the post-genomic era as a valuable discipline to process the protein target information being deciphered. The field plays a crucial role in assigning function to sequenced proteins, defining pathways in which the targets are involved, and understanding structure-function relationships of the protein targets. A key component of this research sector is accessing the threedimensional structures of protein targets by both experimental and theoretical methods. This then leads to the question of how to store, retrieve, and manipulate vast amounts of sequence (1-D) and structural (3-D) information in a relational format so that extensive data analysis can be achieved. We at SBI have addressed both of these fundamental requirements of structural proteomics. We have developed an extensive collection of three-dimensional protein structures from sequence data and have implemented a relational architecture for data management. In this article we will discuss our approaches to structural proteomics and the tools that life science researchers can use in their discovery efforts.

\section{INTRODUCTION}

With the mapping of the human genome largely complete, researchers have begun to focus their attention on gene products and protein function. Approximately 35000 genes have been mapped for the human genome alone, and it has been estimated that each gene is likely to encode for more than one protein. Thus, technologies will have to shift to a high-throughput mode while still focusing on generating quality data to characterize three-dimensional (3-D) structures from the primary amino acid sequences. In fact, there are many privately and publicly funded institutions, collectively termed structural genomics initiatives, which aim to characterize protein structural information using both computational and experimental approaches. If these initiatives succeed, the next bottleneck that researchers have to confront is the storage and retrieval of such data within a discovery environment. There has been a tremendous increase in the availability of bioinformatics databases to the scientific com munity. However, much of the data available to the public are inadequately curated and are often duplicated. The data formats are so divergent that they pose considerable challenges in data integration and manipulation. For any meaningful application of structural proteomics in a drug discovery environment, the structural data generated should be of high quality and formatted for easy access, manipulation, and analysis.

The focus at SBI is on generating large-scale, highly refined 3-D models of protein targets from primary amino acid sequences. Protein structural models assembled in the proprietary database ProMax ${ }^{\mathrm{TM}}$ are constructed using knowledge-based methods augmented by energy-based techniques known as augmented homology modeling. The 3-D models comprehensively cover many superfamilies (in excess of 450 at the time of this writing). Each protein in the database is a dynamically refined computer-generated model that has undergone stringent quality assurance tests and validation based on stereochemical and energetic rules derived from X-ray crystal structures. Extensive analyses of structural features are carried out for each model before it is deposited into the StructureBank ${ }^{\mathrm{TM}}$ — a database management system that has been checked using more than 300 types of tests for accuracy and relational integrity. The system was built on industry-strength relational database management systems Oracle ${ }^{\mathrm{TM}}$ and IBM Universal Database DB2 ${ }^{\mathrm{TM}}$. ProMax has a graphical interface that allows the user to view, interact with, and analyze the 3-D structures, as well as inspect the quality controls associated with each of the models.

\section{THE CURRENT STATUS OF PROTEIN STRUCTURE DETERMINATIONS}

Historically, the worldwide pharmaceutical industry has directed its entire research effort at approximately 500 protein targets. These efforts have produced most of the drugs currently being marketed or that are in the development pipeline. Within the next five years, however, the Human Genome Project and other academic and commercial initiatives are expected to identify several thousand more potential drug targets from among the 35000 or so genes believed to compose the human genome. The genes for thousands of additional targets are also being identified from elucidation of the genomes of pathogenic organisms such as bac- 
teria, viruses, and fungi. Additionally, genetic sequences of thousands of protein targets from other pharmacologically relevant species, such as the rat, mouse, dog, and certain primates, which correlate with relevant human targets are being determined for use in selecting appropriate animal models. Finally, genetic sequences of selected targets from genomic research species such as Caenorhabditis elegans (nematode), Drosophila (fruit fly), Brachydanio rerio (zebra fish), and yeast will also be important for use in gene function identification studies and comparative genomics.

Genes themselves are not drug targets. Rather, they encode proteins, the molecules in the body that directly determine biological function and activities in living organisms and constitute the point of physiological intervention for drugs. Proteins have complex 3-D structures with multiple dynamic properties. Because drugs can affect the physiological and biological activity of a protein by altering its stereochemical properties, determination of a protein structure is critical to the advancement and efficiency of structure-based drug design processes as well as protein structure-function studies.

While current physical methods for determining protein structure, such as nuclear magnetic resonance (NMR) spectroscopy and X-ray crystallography have made great advances in recent years, these techniques are costly and painstakingly laborious, typically requiring one to three years per protein target-a highly impractical timeframe given the burgeoning number of genomic targets and the competitive nature of the pharmaceutical industry. Hence there have been a number of initiatives to take the structure determinations by these physical methods both by private companies and governmental grants under their structural genomics initiatives. The goals for these initiatives are multifold but can be broadly classified as high-throughput protein production, crystallization, and structure elucidation (6). However, the exact outcome of these high-throughput initiatives will not be known for a few more years; there are a number of biological issues to sort out to produce a quantity of protein suitable for crystallization and in the quality of the crystals necessary for good X-ray diffraction data.

In parallel with the experimental methods, there have been great advances in the computational derivation of protein 3-D structures from primary amino acid sequence data. This has

\section{Augmented Homology Modeling Procedure}

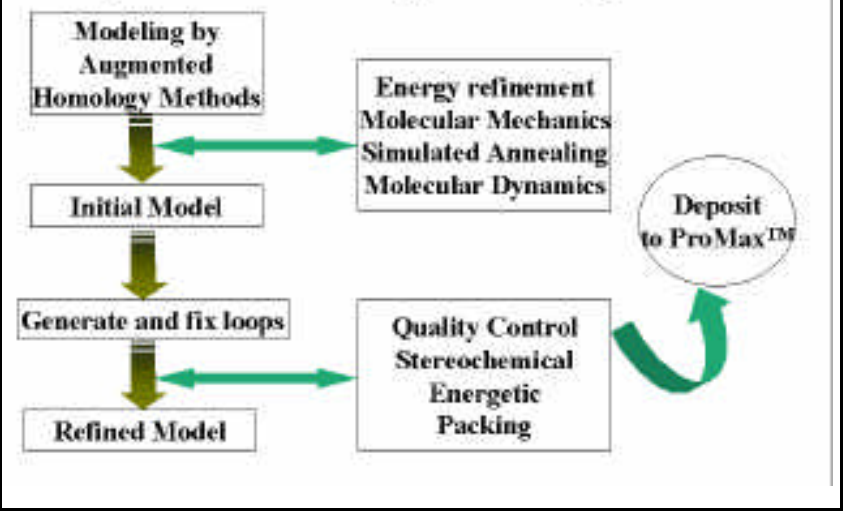

Figure 1. Flow chart of augmented homology modeling procedure. been facilitated by the many years of accumulation and the availability in the Protein Data Bank (PDB) of a large number of experimental structural data (from about 16000 structures) from many unique protein classes. Knowledge of protein fold and classes, in conjunction with other advances in secondary structure prediction, and protein modeling technologies can be used to predict 3-D structures of new proteins from their amino acid information. Even though the prediction of protein structure from the amino acid sequence is viewed with some amount of pessimism, progress has been made in structure predictions as evidenced in the structure prediction contests being held every other year in Asilomar, CA, known as the CASP competitions (http://PredictionCenter.llnl.gov/). The CASP competitions compare the quality of protein structures generated using com putational methodologies to those obtained through NMR and X-ray crystallography. This is a "blind" competition in which only the organizers (and the researchers who provide the experimental structures) know the folds of the protein target sequences submitted for prediction. Over 100 groups and individuals from around the world, including most of the leading academic protein structure laboratories, participate in the com petition, submitting several thousands of protein model entries. The broad categories assessed for accuracy here are comparative or homology modeling, fold prediction, and ab initio modeling. The most advanced and mature methodology happens to be the comparative modeling category in which protein models are constructed based on sequence and structural identity to a known structural template derived from either NMR or X-ray crystallographic methods. At SBI, we routinely exercise the comparative modeling methodology called augmented homology modeling to generate several protein structures at atomic resolution for many biological and pharmaceutical applications.

\section{Augmented Homology Modeling Method}

The world's current output of crystal structures is approximately 3000 for the year 2001, which is just a few hundred more than what was output in the years 2000 and 1999 (Table 1). Even the most enthusiastic proponents of X-ray crystallography do not consider it feasible to solve the structures of the many thousands of proteins that make up the human genome and other organisms in a commercially relevant timeframe. SBI's proprietary computational protein structure homology modeling technologies have helped us overcome this bottleneck by developing 3-D structures in a reasonable timeframe for proteins for which 3-D templates are available. This methodology thus provides access to a significantly greater portion of the protein structures within a practical timeframe.

From public domain sources, we download the primary amino acid sequences of proteins, which are then matched using a variety of sequence alignment methods to find the best structural template(s) to build the initial model. The alignments are then adjusted using information on the secondary structure predictions (8), functional residues, and disulfide bonds. Depending on the quality of the loops in the initial model, the loops may be stripped and reconstructed (4). This initial model, com plete with all loops, is then subjected to refinement procedures, which are based on molecular mechanics, molecular dynamics, and simulated annealing methods (see Figure 1). After each re- 
Table 1. The Growth of Protein Structures in the Protein Data Bank (Including Experimental and Theoretical Models)

\begin{tabular}{|c|c|c|}
\hline Year & $\begin{array}{l}\text { Structures } \\
\text { Deposited }\end{array}$ & $\begin{array}{c}\text { Total Structures } \\
\text { Accessible }\end{array}$ \\
\hline 1972 & 2 & 1 \\
\hline 1973 & 10 & 6 \\
\hline 1974 & 7 & 9 \\
\hline 1975 & 18 & 14 \\
\hline 1976 & 47 & 58 \\
\hline 1977 & 27 & 85 \\
\hline 1978 & 26 & 104 \\
\hline 1979 & 32 & 128 \\
\hline 1980 & 20 & 150 \\
\hline 1981 & 39 & 168 \\
\hline 1982 & 59 & 197 \\
\hline 1983 & 27 & 234 \\
\hline 1984 & 36 & 258 \\
\hline 1985 & 27 & 280 \\
\hline 1986 & 29 & 296 \\
\hline 1987 & 61 & 318 \\
\hline 1988 & 116 & 382 \\
\hline 1989 & 160 & 459 \\
\hline 1990 & 249 & 597 \\
\hline 1991 & 428 & 790 \\
\hline 1992 & 567 & 1006 \\
\hline 1993 & 792 & 1727 \\
\hline 1994 & 1059 & 3091 \\
\hline 1995 & 1221 & 4056 \\
\hline 1996 & 1441 & 5240 \\
\hline 1997 & 1862 & 6833 \\
\hline 1998 & 2178 & 8942 \\
\hline 1999 & 2653 & 11364 \\
\hline 2000 & 2996 & 14062 \\
\hline 2001 & 3035 & 16762 \\
\hline \multicolumn{3}{|c|}{$\begin{array}{l}\text { Note:The number of NMR based structures totals to } 2570 \\
\text { (about } 15 \% \text { of the total). Number of structure released per } \\
\text { day based on } 365 \text { days is } 9 . \text { Some of the newly deposited } \\
\text { structures every year are actually based on improved } \\
\text { refinements of already deposited structures. } \\
\text { Source: http://www.rcsb.org/pdb/holdings/table.html }\end{array}$} \\
\hline
\end{tabular}

finement stage, the model is checked for quality using various quality control procedures. Once the model has passed the quality control processes, it satisfies not only stereochemical criteria but also energetic and packing considerations. The final model is then deposited into the ProMax protein database.

The following problems may arise when homology models are not built carefully or when they are built using fully automated methods:
- Consecutive $\mathcal{Q}$ atoms are farther apart than the optimum distance of 3.7 to $3.9 \AA$, resulting in chain breaks

- Distorted bond lengths and bond angles

- Incorrect isomerization of the peptide backbone in nonproline residues

- Disallowed backbone and side-chain conformations

Our refinement procedures enable us to do the following:

- Remove distortions of covalent geometry by using energetic methods

- Convert disallowed backbone and side-chain conformations into allowed ones using simulated annealing procedures

- Conserve protein core structure and secondary structural elements built by homology

- Rebuild unnatural loop conformations

- Quality control to satisfy energetic and packing considerations

\section{COMPARISON WITH PUBLISHED X-RAY STRUCTURES}

We searched and downloaded from the PDB recently deposited NMR and X-ray crystal structures of proteins for which we had already predicted 3D structures from our augmented homology modeling methods. Our predicted models were determined prior to the release of experimental structures.

We have shown in Figure 2 the breakdown of the root mean square derivation (RMSD) between the experimental structure and our model by means of a pie chart. The sequence identity

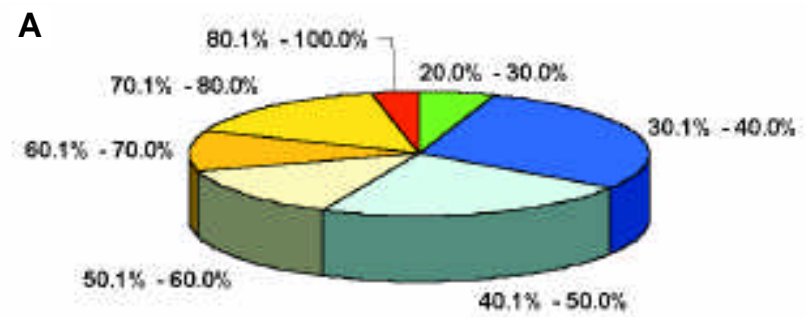

B



Figure 2. Comparison of SBI models with published X-ray structures showing (A) the root mean square deviation of the protein structural core, and (B) percentage sequence identity of templates used in model building. 
range of the templates used for model building is also indicated in the figure. Clearly, when a highly iterative protein modeling scheme is used, errors in the model constructions are low and one is able to generate more accurate models of good quality. The accuracy is also seen to be reflective of the sequence identity of the templates chosen.

\section{PROTEIN STRUCTURE ORGANIZATION BY MEANS OF A RELATIONAL DATABASE FORMAT}

If the output of the high-throughput protein structural information has to be used in a discovery environment, it is essential to store it in a network-oriented information management system for scalability, ease of access, and extensive data mining capabilities. When designing our protein structure database management system StructureBank, our primary goal was to build a reliable decision support system with analytical processing and data mining capabilities that allowed our internet-based subscribers to access our growing database of protein structures and extract correlations buried within this data.

Recently, initiatives to standardize information representation and data interchange have gained importance in life sciences (3). Extensible markup language (XML) has become the fastest-growing means of transferring data across disparate platforms in many fields and has been proposed for adoption by the bioinformatics community (1). StructureBank was built using XML standard and provides access to thousands of proprietary protein structures over the web from a variety of clients, including servers that consume XML.
StructureBank was developed to manage ProMax, a collection of proprietary models of proteins that play crucial roles in various diseases such as cancer, diabetes, and AIDS. These proteins have been partitioned into families by function. In addition to atomic coordinates, ProMax contains information about alignment, secondary structure predictions, quality control results, and tools to visualize the models in different formats.

Furthermore, every model in the ProMax collection has structure comparison tools with all other models at different domain sizes. These comparisons were made possible by using The $\mathrm{Na}-$ tional Center for Biotechnology Information (NCBI)'s VAST (Vector Alignment Structure Tool) (5). This method generates and uses vector alignment of the secondary structure elements of any two proteins to produce similarity regions at various domain levels. For every model, along with similarity rankings with all other models, we also store their superposition matrices.

Additionally, ProMax contains relevant information shown to be critical in the design of drugs in terms of their interaction with corresponding proteins. Some of these have annotated information on the model's structure with its function and related disease. Examples gathered from the literature include:

- Active/functional site conformations

- Hydrophobic packing and nucleation sites

- Protein flexibility and rigidity regions

- Electrostatic potential

- Surface curvatures

- Exposed (hydrophilic) and buried (hydrophobic) regions

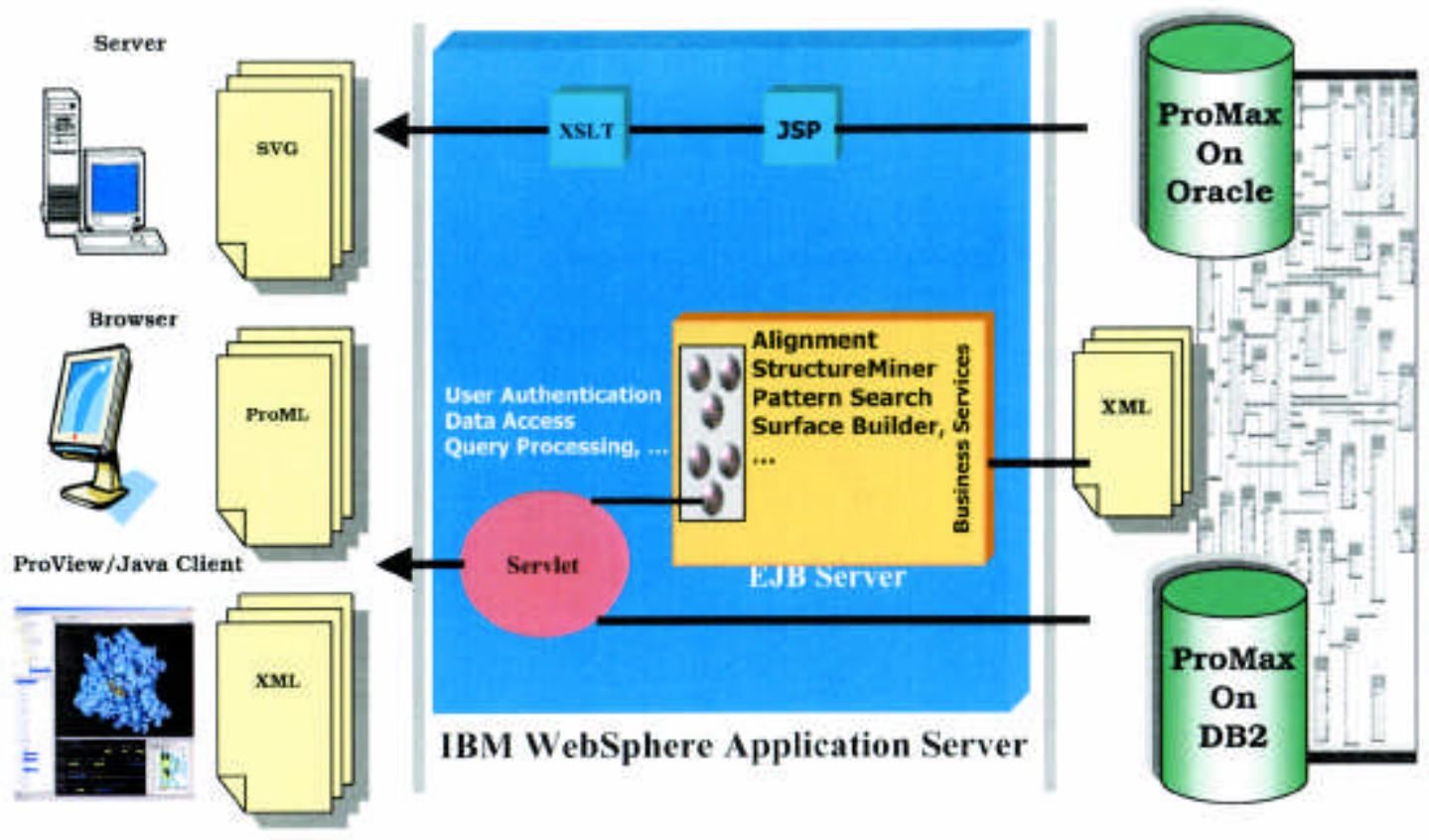

Figure 3. Multi-tier architecture representation of the ProMax protein structural database, which can be extended to store and analyze any type of structural proteomics data. 


\section{STRUCTUREBANK: A RELATIONAL DATABASE ARCHITECTURE TO STORE STRUCTURAL PROTEOMICS DATA}

StructureBank is designed as an $n$-tier distributed system using JAVA. The data layer comprises a relational database as well as XML documents. Together they maintain protein sequences, structures, annotations, and the pertinent information for similarity searches as mentioned in the previous section. This relational database is maintained on Oracle $8 \mathrm{i}^{\mathrm{TM}}$ as well as IBM DB2 ${ }^{\mathrm{TM}}$. The schematic representation of such a database is given in Figure 3.

The middle tier is built using JAVA Servlets, Enterprise JAVA Beans (EJBs), and JAVA Server Pages and sends requested protein information in the form of XML documents that are based on ProML, a protein description schema developed by us. ProML draws on many ongoing efforts in standardization of sequence and protein related information using XML. These include bioXML work at the Institute for Biomedical Computing and Washington University in St. Louis, MO, USA.

Some of the middle-tier business services, which are modeled through interaction with protein scientists and structural biologists, are similarity searched within the proteins for sequence, structure and environment, surface generation, and sequence pattern matching.

Examples of these comparisons include:

- Given an amino acid sequence and a selected group of proteins within the database, the sequence similarity component finds and ranks homologous protein sequences spanning from a family to the entire database.

- The StructureMiner similarity component compares two structures for preferred environment calculated within a shell of each residue in a protein. This reduced-variable representation approach developed at SBI (7) uses the Chemical Neighborhood matrix for comparisons between proteins and preserves the chemical and functional information of each amino acid by transferring 3-D information to 1-D. The spatial environment matrix for twenty amino acid residues for a particular protein is compared and correlated with computed values of other proteins to get a relative sim ilarity index. The comparison is improved by using stacks of Chemical Neighborhood matrices.

StructureBank is accessible over the Internet or the Intranet through ProView. This graphical user interface, written as an applet, runs inside Netscape ${ }^{\mathrm{TM}}$ browser or Internet Explorer ${ }^{\mathrm{TM}}$ and presents sophisticated 3-D visualization of protein structures. Furthermore it offers various sequence-based and structure-based searches. The ProView applet sends XML messages to servlets deployed on the application server and gets another XML message back in response. This way each side of the connection works with a fully parsed Document Object model of the XML.

\section{CONCLUSIONS AND PERSPECTIVES}

At a time when technologies are needed to process vast amounts of post-genomic data in a high-throughput fashion, structural proteomics plays a major role by characterizing the genomic information as 3-D structures accessible to life science researchers in the discovery field.
While high-throughput X-ray and NMR experimental structure determination methods hold much promise, until such time that we see actual results, one has to resort to validated computational approaches such as augmented homology modeling to access 3-D protein structures. The efficiencies in speed and cost have to be achieved using robust $3-\mathrm{D}$ protein modeling approaches and good information management systems. To integrate both the bioinformatics and the 3-D structural data of the proteins, we have developed the StructureBank system, which readily puts high-quality protein models in researchers' desktop computers. Further technological advances will be made in computer algorithms to push the protein structure prediction to the next level as evidenced by the progress of "new fold" predictions in the CASP competitions. This advance will also be met with equally robust tools to do data mining from protein structures so that life science researchers can perform a number of experiments in silico by asking a series of questions and generating hypotheses, which can be confirmed later by experiments (2). On the integration side, we will also be seeing the linking of pathways data, proteomics analysis data (like 2-D gel analysis), and small molecule data in one common platform. In fact, in the near future, biologists, chemists, and computational chemists will be looking at one common platform to derive useful information to speed up their research efforts.

\section{REFERENCES}

1.Achard, F., G. Vaysseix, and E. Barillot. 2001. XML, bioinformatics and data integration. Bioinformatics 17:115-125.

2.Billingsley, F., V. Karlov, and C. Padilla. 2001. Putting a rocket under com puting for life sciences. Scientific Computing World, July/August, 2001.

3.Brazma, A. 2001. On the importance of standardization in life sciences. Bioinformatics 17:113-114.

4.Dudek, M.J., K. Ramnarayan, and J.W. Ponder. 1998. Protein structure prediction using a combination of sequence homology and global energy minimization. J. Comp. Chem. 19:548-573.

5.Gibrat, J.-F., T. Madeg, and S.H. Bryant. 1996. Surprising similarities in structure comparison. Curr. Opin. Struct. Biol. 6:377-383.

6.Jhoti, H. 2001. High-throughput structural proteomics using $\mathrm{x}$-rays. Trends Biotechnol. (Suppl.) 19:S67-S71.

7.Karlov, V., D. Beglov, E. Koistinen, D. Timotijevic, C. Padilla, J. Zheng, and K. Ramnarayan. 2001. Automated mining of protein structural similarities using reduced variable representation. 221st ACS Meeting, San Diego, CA, April 4, 2001

8.Petersen, T.N., C. Lundegaard, M. Nielsen, H. Bohr, J. Bohr, S. Brunak, G.P. Gippert, and O. Lund. 2000. Prediction of protein secondary structure at $80 \%$ accuracy. Proteins $41: 17-20$.

Address all correspondence to:

Kal Ramnarayan

Structural Bioinformatics

10929 Technology Place

San Diego, CA 92127, USA

e-mail:kalram@strubix.com 